American Journal of Pharmacology and Toxicology 2 (2): 80-88, 2007

ISSN 1557-4962

(C) 2007 Science Publications

\title{
Curcuma longa, Glycyrrhiza glabra and Moringa oleifera Ameliorate Diclofenac-induced Hepatoxicity in Rats
}

\author{
Alaaeldin A. Hamza \\ U.A.E. University, Al-Ain, P.O. Box: 17551, U.A.E.
}

\begin{abstract}
Although treatment with therapeutic agents may be one of the main causes of liver toxicity, there have been only limited attempts at thorough research efforts to seek solution. Diclofenac (DIC) is a widely used anti-inflammatory drug that causes liver toxicity. This DIC-induced hepatotoxicity was found to be associated with oxidative damage. In this report, extracts of herbal plants that are traditionally used for treating many illnesses were examined, namely Curcuma longa (CL), Glycyrrhiza glabra L (GL) and Moringa oleifera Lam (MO). They all showed a novel hepatoprotective effects against DIC-induced hepatotoxicity in rats. Administration of DIC at $150 \mathrm{mg} / \mathrm{kg}$ developed acute hepatic damage, as demonstrated by increased serum alanine aminotransferase (ALT) activity and histopathological changes. In addition, DIC treatment resulted in an increase in the hepatic malonialdehyde level and depletion in total antioxidant capacity, reduced glutathione content, catalase, and superoxide dismutase activities. Treatment with herbal extracts for 30 days before DIC treatment significantly ameliorated the indices of hepatotoxicity induced by DIC. In addition, these herbs alleviated DIC-induced oxidative changes in liver. These results suggest that CL, GL, and MO inhibit DIC-induced hepatotoxicity and might serve as a novel combination chemotherapeutic agent with DIC to limit its free radical-induced organ injury.
\end{abstract}

Key words: Diclofenac, Hepatic toxicity, protection, Curcuma; Glycyrrhiza

\section{INTRODUCTION}

Diclofenac (DIC), Voltaren, is a nonsteroidal anti-inflammatory drug, which has analgesic and antiinflammatory effects and widely used for treatment of a variety of rheumatoid disorders ${ }^{[1]}$. DIC causes a rare but potentially sever liver injury in humans ${ }^{[2 ; 3]}$. The hepatotoxicity of DIC was also documented in experimental animal studies ${ }^{[1 ; 4 ; 5]}$.

The mechanism of DIC hepatotoxicity involves covalent protein modification by reactive metabolites ${ }^{[6 ;}$ $7 ; 8]$, oxidative stress generation by peroxidase-catalyzed reaction ${ }^{[9 ; 4]}$ and mitochondrial injury propagation by reactive oxygen species ${ }^{[10 ; 11]}$.This DIC-induced hepatic toxicity was prevented by antioxidants and also cytochrome P-450 inhibitors ${ }^{[12 ; 4]}$. It is logical to consider antioxidants as primary candidates to counteract such toxic effect. In recent years, accumulating evidence supported the protective effects of phenolic antioxidants from medicinal plants against oxidative stress-mediated disorders ${ }^{[13]}$. Although several natural extractions from plants have been shown to protect against chemical-induced liver toxicity, a consensus on the protective effects of natural substances for the treatment of DIC- induced hepatic toxicity however has not yet been reached.

Curcuma longa Linn (CL) (turmeric) rhizome (Zingiberaceae) is one of the most common medicinal plants in tropical areas of Asia and Africa, widely used as a food additive and coloring agent ${ }^{[14]}$. In tropical regions of Asia, CL has been used as a traditional remedy for the treatment of inflammation and other pharmacological effects ${ }^{[15]}$. The beneficial effects of CL have been postulated to be due to, the phenolic yellow pigments of turmeric, curcuminoids with the major compound being curcumin ${ }^{[14]}$. CL and its main component curcumin are found to exhibit different protective effects in animals such as hepatoprotective [16;17; 18; 19], antioxidant ${ }^{[20 ; 14]}$, antiatherogenic ${ }^{[20]}$ and antitumorigenic ${ }^{[21 ; 22]}$ activities.

Glycyrrhiza glabra L (GL) (licorice), a perennial leguminous plant, is the most popular herbal remedy in the Mediterranean and Middle East ${ }^{[23]}$. A number of pharmaceutical effects of GL root are already known including anti-inflammatory, antihepatitis, antivirus, antiulcer and anticarcinogenesis ${ }^{[24 ; 25]}$. The extract of GL and its flavonoids were found to have potent

Corresponding Author: AlaaEldin A.Hamza, Department of Biology, Faculty of Science, U.A.E. University, Al.Ain, UAE, P.O. Box: 17551,Tel: 971- 3 -7132687, Fax: 971- 3- 7671291 
hepatoprotective effect against a variety of hepatotoxic agents including $\mathrm{CCl}_{4}{ }^{[26 ; 27]}$, and aflatoxin B1 ${ }^{[28]}$. Constituents of licorice include triterpenoids, such as glycyrrhizin and its aglycone glycyrrhizic acid, and various polyphenols such as licochalcone $\mathrm{A}$ and glabridin ${ }^{[29 ; 24 ; 30]}$. Seven constituents, with antioxidant capacity were isolated from Glycyrrhiza glabra. Among these compounds, Glabridin constituted the major amount of the crude extract $(11.6 \%$,w/w) and was the most potent antioxidant ${ }^{[29]}$.

Moringa oleifera Lam (MO) (horse radish tree) (Moringaceae) is a small sized tree, which is native to south Asia and also grows in tropical Africa ${ }^{[31]}$.Various parts of MO are generally known for their multiple pharmacological effects including their antiinflammatory [32], antitumor [10; 33; 34] and antihyperglycemic ${ }^{[35]}$ effects. The extract of MO has been found to have potent antioxidant action in vivo ${ }^{[36]}$ and in vitro ${ }^{[37]}$ studies. Phytochemical investigations isolated the bioactive compounds from the seeds of MO , and found to have glycosides such as four (alpha-Lrhamnosyloxy-benzylglucosinolate), niazimicin and niazirin, beta-sitosterol and $41 \%$ moringa oil (which was found to contain high level of unsaturated fatty acids similar to olive oil) ${ }^{[38 ; 33 ; 39 ; 40]}$.

The main objective of this investigation is to evaluate the protective effects of CL, GL, and MO against DIC-induced hepatic toxicity in male rats. Liver toxicity was induced by administrating a single DIC dose in the presence or absence of plants. This study includes biomarkers of hepatic toxicity such as histopatological study and serum activity of ALT. Oxidative stress and antioxidants of liver will study by determination of reduced glutathione (GSH), superoxide dismutase (SOD) and catalase (CAT) enzyme activities, lipid peroxidation (malondialdehyde, MDA) and FRAP assay.

\section{MATERIALS AND METHODS}

Chemicals: The dried plants, CL rhizome, GL root, and MO seeds were purchased from herbal local store at Cairo, Egypt. DIC (Voltaren) was purchased from Novartis Pharma AG Company, Swizererland. Thiobarbituric acid, reduced glutathione, 5,5-dithiobis (2-nitrobenzoic acid), Folin's reagent, epinephrine, SOD enzyme, $\mathrm{H} 2 \mathrm{O} 2$, and bovine albumin were purchased from Sigma Chemical Co. (St. Louis, MO). All other chemicals were purchased from common commercial suppliers.

Animals: Adult male albino rats (150-200g) of the Sprague-Dawley strain were obtained from the Animal
House of the National Organization of Drug Control and Research, Egypt. They were maintained on standard pellet diet and tap water ad libitum. Rats were kept in polycarbonate cages with wood chip bedding under a $12 \mathrm{hr}$ light/dark cycle and room temperature 22$24^{\circ} \mathrm{C}$. They were acclimatized to the environment for two week prior to experimental use. This study was performed according to the Local Animal Ethics Committee Guidelines for Animal Experimentation.

Extraction: To increase the yield of extraction in a shorter time and at a lower temperature, the liquidphase microwave-assisted process was used for extractions of CL, GL and MO according to the method described by Pan et al., [41]. This microwave-assisted extraction application is based on the selective heating of the matrix that contains the target extract when the matrix is immersed in a solvent such as ethanol and water, which is transparent to the microwaves. This solvent, allows for selective heating of particular components within the materials being treated, without using excessive energy. Every $10 \mathrm{gm}$ of ground-dried plants was mixed with $100 \mathrm{ml}$ of $70 \%$ ethanol in 250 $\mathrm{ml}$ conical flask. Mixtures were then irradiated with microwave for two minutes. The output power of the microwave oven is $300 \mathrm{~W}$. The suspensions were irradiated for 25 seconds with power on to give the desired temperature of about $80{ }^{\circ} \mathrm{C}$ and then for 5 second with power on for heating and then for 10 second with power off for cooling. Then the extracts were finally filtered through gauze and evaporated under vacuum at $40^{\circ} \mathrm{C}$ using a rotary evaporator.

Treatment Regime: DIC reagent was light-protected and was prepared in a saline solution and given in a volume of one $\mathrm{ml} / 100 \mathrm{~g}$ body weight. Control animals received an equivalent volume of saline based on body weight. The three extracts were given orally by gavage at the volume $1 \mathrm{gm} / \mathrm{Kg}$ of body weight. Rats were randomly divided into eight groups $(n=8)$ and were subjected to the following treatments: Control group received daily an equivalent volume of $1 \%$ DMSO. DIC-treated group received a single intraperitoneal dose of DIC (150 mg/kg b.wt.). The hepatotoxic dose of DIC was based on previous hepatotoxic studies of DIC in rodents $[1 ; 5]$. Rats of the three following groups were orally administered with one of the three plant extracts for three 30 days. Rats of the last three groups were orally pretreated with one of the three extracts daily for 30 days and then one hour after extract treatment the rats were give a single dose of DIC injection. After 24 hours of DIC treatment, herbal extract or vehicle solution administration, blood and organ samples were collected from all eight groups. 
Sample Preparation: Following diethyl ether anesthesia, blood was collected from the retro-orbital plexus [42]. Following sacrifice, the same lobe of the liver was removed and immediately immersed in $10 \%$ buffered formalin, $\mathrm{pH}$ 7.4, for histopathological examinations. For other biochemical examinations, other liver samples were removed and homogenized in ice-cold buffered solution $(150 \mathrm{mM} \mathrm{KCl}, 50 \mathrm{mM}$ Tris, $\mathrm{pH}$ 7.4). The ratio of tissue weight to homogenization buffer was 1:10. The crude extract was aliquoted and stored at $-20^{\circ} \mathrm{C}$. From the latter, suitable dilutions for determination of the levels of GSH, LP product MDA, total proteins, and activities of SOD and CAT were prepared in suitable different buffers. To obtain serum, blood was collected and then centrifuged at 3000 r.p.m. for 20 minutes in a refrigerated $\left(4^{\circ} \mathrm{C}\right)$ centrifuge. Fresh serum samples were used for the determination of albumin and ALT enzyme activities.

Biochemical Assays and Histopathology : The GSH content in the liver homogenate was determined using the method described by Van Dooran et al [43]. The basis of the GSH determination method is the reaction of Ellman's reagent 5,5-dithiobis (2-nitrobenzoic acid) (DTNB) with thiol group of GSH at $\mathrm{pH} 8.0$ to give yellow color of 5-thiol-2- nitrobenzoate anion.

MDA is the most abundant individual aldehyde resulting from lipid peroxidation (LP) breakdown in biological systems and is used as an indirect index of LP [44]. Determination of MDA in biological materials is based on its reaction with thiobarbituric acid to form a pink complex with absorption maximum at $535 \mathrm{~nm}$ [45].

The activity of SOD enzyme in liver homogenate was determined according to the method described by Sun \& Zigman [46]. This method is based on the ability of SOD to inhibit the auto-oxidation of epinephrine at alkaline $\mathrm{pH}$ to adrenochrome and other derivatives, which are easily monitored in the near-UV region of the absorption spectrum. CAT activity was determined by measuring the exponential disappearance of $\mathrm{H} 2 \mathrm{O} 2$ at $240 \mathrm{~nm}$ and expressed as units/mg of protein as described by Aebi [47].

The total antioxidant capacity in liver was evaluated using ferric reducing antioxidant power (FRAP) assay. The FRAP assay was determined according to the method described by Benzie and Strain [48]. The FRAP assay measures the change in absorbance at $593 \mathrm{~nm}$ due to the formation of a blue colored ferroustripyridyltriazine complex from colorless oxidized ferric form by the action of electron donating antioxidants.

ALT activity was determined in serum according to the method described by Reitman \& Frankel [49]. The assay was performed using Bio-Merieux reagent kits and following their instruction manual. The total protein content in liver was determined according to the Lowry's method modified by Peterson, [50]. In all the estimations, absorbance was recorded using a Shimadzu recording spectrophotometer UV-160A.

For the histological examination, small pieces of liver were fixed in $10 \%$ neutral phosphate-buffer formalin and the hydrated tissue sections $(5 \mu \mathrm{m}$ in thickness) were stained with Hematoxylin and Eosin. These sections were then examined under a Leica DMRB/E light microscope.

Statistical Analysis: The results are expressed as group mean \pm SE. The statistical analysis was carried out using one-way analysis of variance (ANOVA), with SPSS version 10 statistical program (SPSS Inc., Chicago, IL, USA). ANOVA performed to detect differences between all various groups. When significant differences by ANOVA were detected, analysis of a difference between the means of the treated and control groups were performed using Dennett's t test.

\section{RESULTS}

Effect tested extracts on DIC-induced liver injury: After treatment with DIC, level of serum ALT was significantly $(\mathrm{P}<0.001)$ increased compared to the control by about $208 \%$ (Fig. 1). Rat receiving extract of any of the three herbs used in this study (CL, GL, or MO) did not show any significant change the serum marker of liver damage. While pretreatment with any of the three herbs attenuated the DIC-induced elevation of serum ALT activity. In compared to DIC group, a significant decrease in ALT level $(\mathrm{P}<0.001$ with $\mathrm{CL}$, $\mathrm{P}<0.01$ with GL, $\mathrm{P}<0.001$ with $\mathrm{MO}$ ) was observed in rats pretreated with tested herbal extracts. The increase of ALT level was reduced by about $47 \%, 43 \%$, and $27 \%$ after pretreatment with MO, CL, or GL respectively.

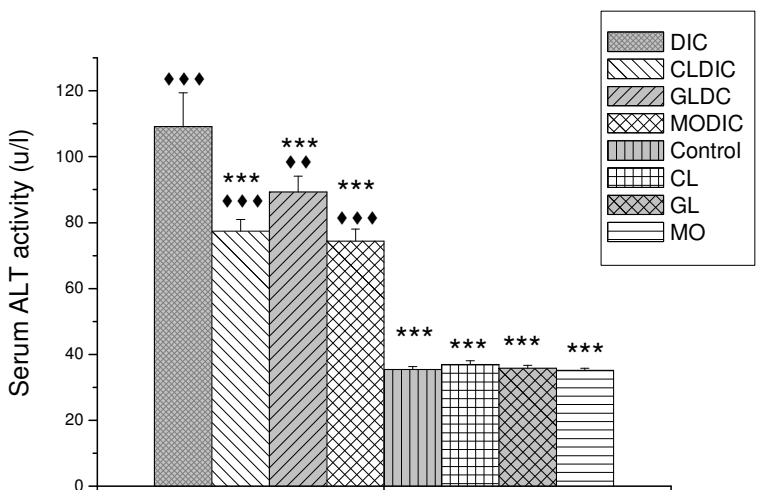

Fig. 1. Effect of CL, GL, or MO on serum ALT activity in control and DIC-treated rats. Columns represent the means \pm S.E.M $(\mathrm{n}=8) . \diamond{ }_{\mathrm{P}<0.001}$ vs. control group. $* * * \mathrm{P}<0.001$ vs. DIC-treated rats. 
Effect of tested extracts on liver lipid peroxidation: The effect of tested herbal plants on the DIC-induced LP was examined through monitoring the levels of MDA. Hepatic MDA shows significant increments $(\mathrm{P}<0.001)$ in its mean value by about $66 \%$, following DIC administration (Fig. 2). None of the three tested herbal plants alone had an effect on hepatic MDA level with respect to control group. However, pretreatment with any of the three plants completely reverses the DIC-induced elevation of hepatic MDA level. In case of GL and MO, The protection against the elevation of MDA was $100 \%$. Protection with CL was only $85 \%$ (Fig. 2).

Role of herbal plants on hepatic total antioxidant capacity: DIC treatment induced a significant decrease $(\mathrm{P}<0.001)$ in hepatic FRAP content by about $17 \%$. While the pretreatment with CL, GL or MO was prevented this decrement in its mean value by about $87 \%, 58 \%$ and $91 \%$ respectively. However, in rats pretreated with GL before DIC treatment, the mean value of FRAP was still lower $(\mathrm{P}<0.05)$ than the mean value of control (Fig. 3).

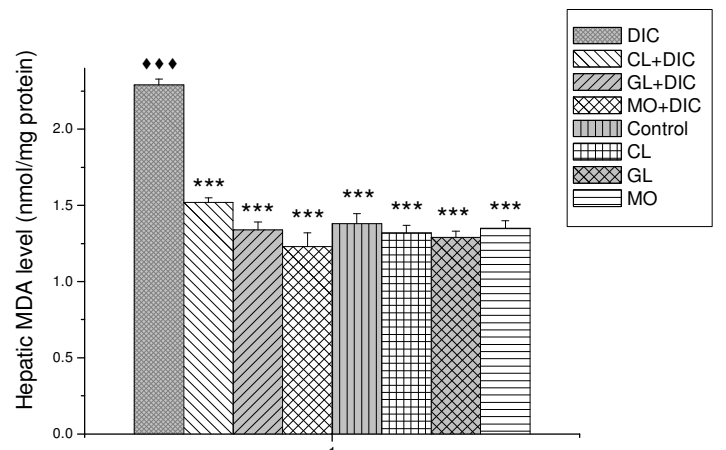

Fig. 2. Effect of CL, GL, or MO on hepatic MDA level in control and DIC-treated rats. Columns represent the means \pm S.E.M $(\mathrm{n}=8) . \diamond \mathrm{P}<0.001$ vs. control group. $* * * \mathrm{P}<0.001$ vs. DIC-treated rats.

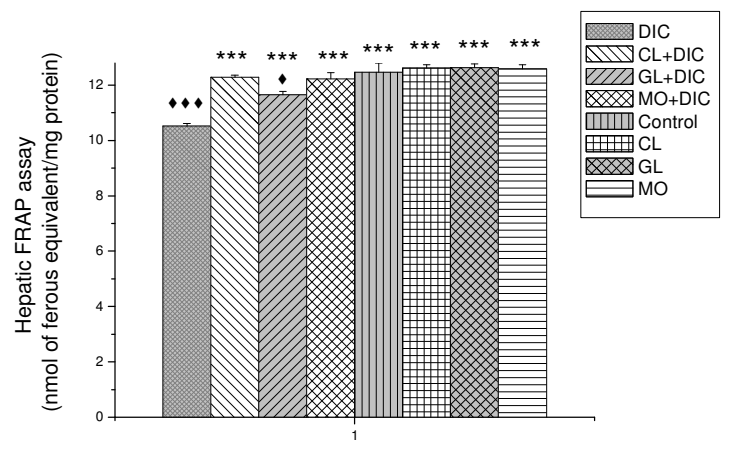

Fig. 3. Effect of CL, GL, or MO on hepatic FRAP assay in control and DIC-treated rats. Columns represent the means \pm S.E.M $(\mathrm{n}=8) . \mathrm{P}<0.05, \diamond \mathrm{P}<0.001$ vs. control group. $* * * \mathrm{P}<$ 0.001 vs. DIC-treated rats.
Role of herbal plants on antioxidants: In DIC-treated rats a significant decrease $(\mathrm{P}<0.01,68.33 \%$ of control $)$ in GSH level was observed in liver 24 hours post treatment (Fig. 4). Pretreatment with either CL or MO attenuated DIC-induced decrease of GSH by about $85 \%$ and $19 \%$ respectively. GL administration prior to DIC treatment induced a significant increase $(\mathrm{P}<0.05)$ in the GSH level by about $29 \%$ compared to control. While treatment with either GL or MO alone did not induce any effect in the level of GSH mean value, treatment with $\mathrm{CL}$ alone induced a remarkable increase in the level of GSH.

DIC-treated rats showed a significant decrease $(\mathrm{P}<0.001)$ in hepatic CAT activity by about $21 \%$ (Fig. 5a). Significant increase in hepatic CAT activity was however shown in rats pretreated with $\mathrm{CL}(\mathrm{P}<0.001)$, GL $(\mathrm{P}<0.05)$ or $\mathrm{MO} \quad(\mathrm{P}<0.001)$ with respect to corresponding group treated with DIC alone. The mean value of CAT activity was still significantly lower $(\mathrm{P}<0.001)$ than the corresponding control value in rats pretreated with either CL or GL before DIC treatment.

There was a significant decrease $(\mathrm{P}<0.001)$ in hepatic SOD activity by about $24 \%$ in DIC treated rats (Fig.5b). Pretreatment with any of the tested extracts ( $\mathrm{CL}, \mathrm{GL}$ or $\mathrm{MO}$ ), prevented the decrements in its mean value by about $60 \%, 55 \%$, and $95 \%$ respectively. These cellular enzymatic antioxidants did not differ significantly from control levels when animals treated with any of the three plants alone.

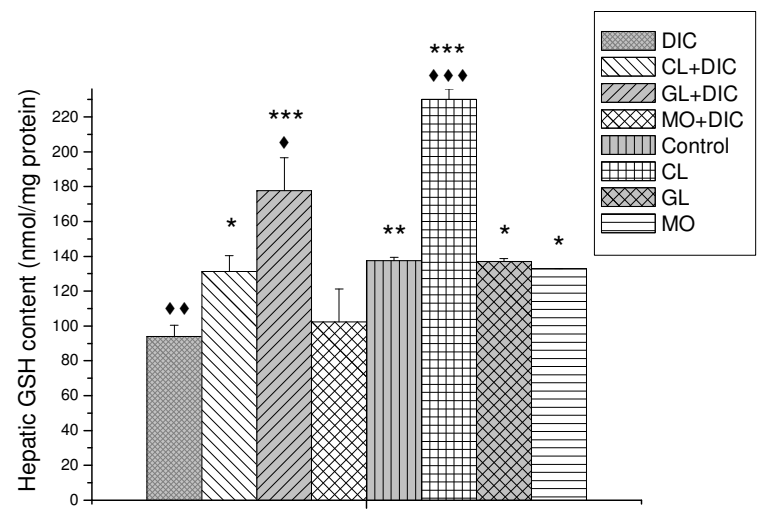

Fig. 4. Effect of CL, GL, or MO on hepatic GSH content in control and DIC-treated rats. Columns represent the means \pm S.E.M

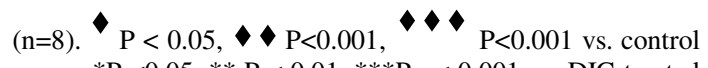
group. $* \mathrm{P}<0.05, * * \mathrm{P}<0.01, * * * \mathrm{P}<0.001$ vs. DIC-treated rats.

Effect of Herbal plants on histolopathological changes: Hepatocytes from DIC-treated rats displayed 
clear cellular degeneration and loss of the distinct liver characteristic configuration (Fig. 6 a \& c). Dilatation of the central vein, enlargement in portal areas observed. Severe necrosis observed in the portal and central areas of the lobule. The marked necrobiotic changes in the liver were mainly in the form of degenerated cells and some cells have eosinophilic cytoplasm with pyknosis of nuclei. Histopathological changes in the liver included also congestion in central veins, portal vessels, and sinusoids. Infiltration of mixed inflammatory cells observed around the necrotic hepatocytes and in portal area. Interestingly, treatment with $\mathrm{CL}, \mathrm{Gl}$, and MO prevented the necrotic changes along with histopathological changes induced by DIC treatment. The liver appears normal except for few scattered degenerated cells (Fig. $7 \& 8$ ).

\section{DISCUSSION}

The aim of this investigation was to study the protective effects of CL, GL, and MO on DIC-induced liver toxicity in rats. To my knowledge, this is the first study to evaluate the protective effects of CL, GL, and MO crude extracts against liver damage induced by DIC in experimental animals. The hepatotoxicity effects of DIC in both humans and experimental animals have been well-documented [3; 1; 4].

In agreement with a previous study [5], the present work showed that intraperitoneally injection of DIC as a single dose $(150 \mathrm{mg} / \mathrm{kg} \mathrm{b} \mathrm{wt})$ induced severe hepatic damage in adult male rats. The DIC-induced hepatotoxicity assessed by significant increments in serum ALT activity and confirmed by histological changes in liver. Most of the hepatocytes in DIC-treated rats displayed cellular degeneration and loss of their characteristic configuration. Moreover, the marked necrobiotic changes in the liver were mainly in the form of degenerated vacuolated cells and karyolysis or pyknosis of nuclei. Histopathological changes in the liver included also dilatation of blood vessels, congestion in the lobules, enlargement of portal areas, and infiltration of mixed inflammatory cells around the necrotic hepatocytes and the portal area.

The DIC-induced liver lesion was associated with massive elevation in liver MDA level. The MDA elevation has been well accepted as a reliable marker of lipid peroxidation [44]. MDA elevation is a result of oxidative stress demonstrated here through the decrease of total antioxidant capacity, GSH level, and antioxidant enzyme activities (SOD \& CAT) in liver. The oxidative stress occurs when the generation of ROS overrides the ability of the endogenous antioxidant system to remove excess ROS [51]. This over productions of ROS subsequently leads to cell damage through oxidation of cell membrane biomolecules such as lipids, proteins and DNA [51]. Similar dose of DIC has been shown to induce massive DNA fragmentations, increase the MDA level and to deplete the levels of GSH and SOD in rat liver [5]. The mechanism of DIC-induced ROS generation has been shown to involve peroxidase-catalyzed production of DIC radicals [9] and cytochrome P450- mediated bioactivation of DIC [4]. DIC, however, found to induced liver damage through various mechanisms such as covalent protein modification by reactive metabolites $[6 ; 7 ; 8]$ and mitochondrial injury $[10 ; 11]$. This DICinduced hepatic toxicity was prevented by antioxidants and also cytochrome P-450 inhibitors [12; 4].

In the present work, pretreatment with CL attenuated the necrotic and the others histopathological changes induced by DIC treatment and partly prevented the elevation in serum ALT activity. These findings are consistent with previous studies, where CL treatment was shown to protect liver from hepatotoxicity induced by aflatoxin [16] or by Carbon tetrachloride (CCL4) $[17 ; 18]$. Moreover, the hepatoprotective effect of the active ingredient of CL, Curcumin, has been shown to prevent both the histological and biochemical changes induced by alcohol in livers of rats [19]. Results from

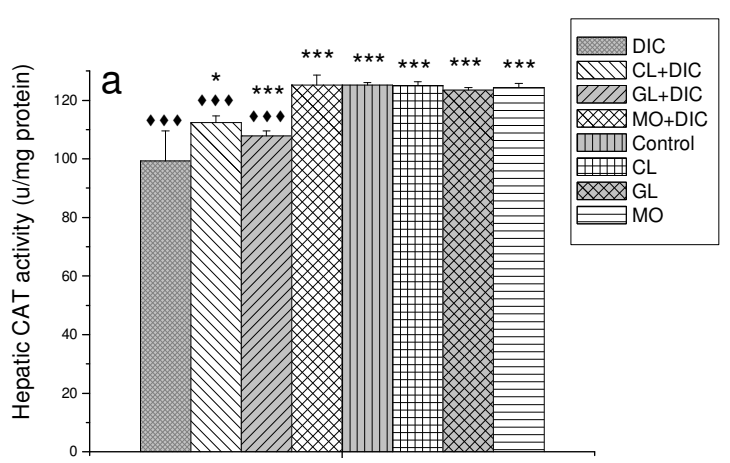




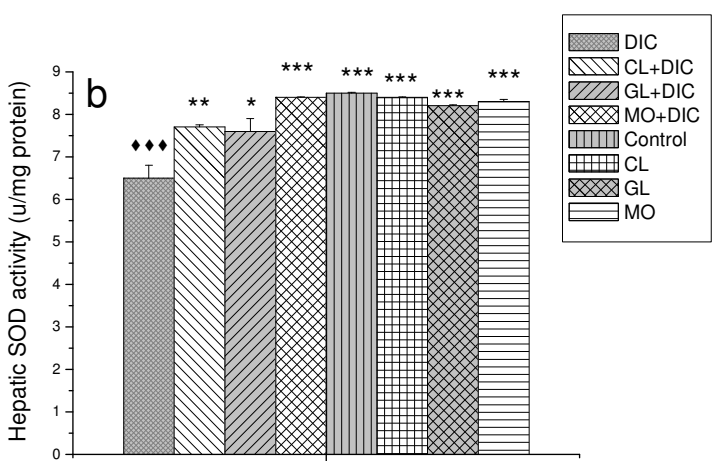

Fig. 5. Effect of CL, GL, or MO on hepatic (a) CAT and (b) SOD activity in control and DIC-treated rats. Columns represent the means \pm S.E.M $(n=8)$ $\checkmark \mathrm{P}<0.001$ vs. control group. $* \mathrm{P}<0.05$, ** $\mathrm{P}<0.01$, *** $\mathrm{P}<0.001$ vs. DIC-treated rats.

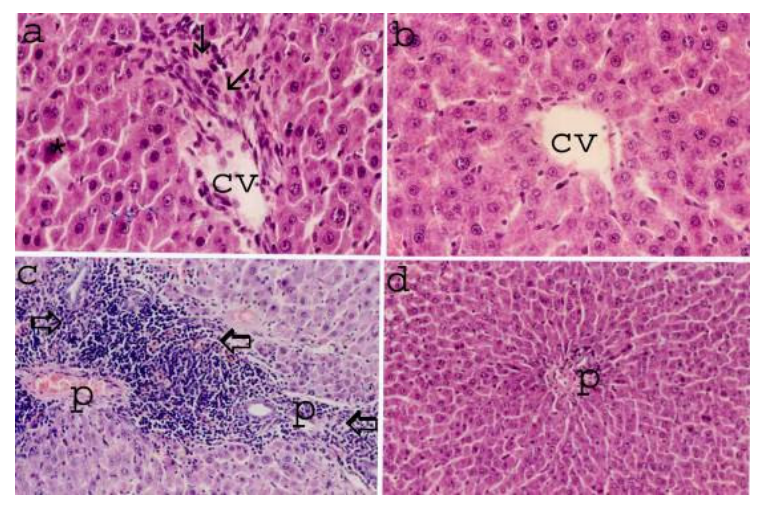

Fig. 6. Light micrographs of liver sections: Liver section from control rats showing normal hepatic architecture $\{(b)$ in central area \& (d) in portal area $\}$.(a) Liver sections from DIC group in central area showing necrosis and degeneration of hepatocytes, congested sinusoids with inflammatory cell infiltration (thin arrows) and some cells with eosinophilic cytoplasm and pyknotic nucleus (*). (c) Liver section from DIC group showing enlargement of portal area, hepatocyte degeneration with inflammatory cell infiltration (thick arrows). P, portal tract; CV, central area; original magnification X 200 in (c \& d) and X 400 in $(\mathrm{a} \& \mathrm{~b}) ; \mathrm{H} \& \mathrm{E}$.

this study suggest that CL exert an antioxidant effect against DIC-induced oxidative stress by attenuating not only MDA elevation, but also the depletion in total antioxidant capacity, GSH, CAT, and SOD. Similarly, $\mathrm{CL}$ has been reported to prevent hepatic MDA elevation [18] and reduce depletion of antioxidant substances [17] in the CCL4 intoxicated rats. Curcumin and its structurally related compounds (Curcuminoids), the phenolic yellowish pigments of $\mathrm{CL}$, were documented as the principal antioxidant constituents of CL $[52 ; 14]$.

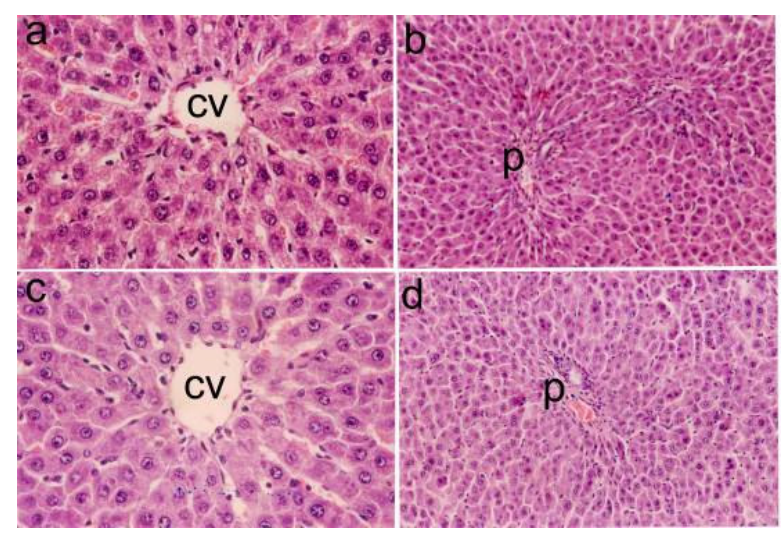

Fig. 7. Light micrographs of liver sections (a-d). (a \& b) Liver section of rat treated with DIC+CL showing normal appearance of hepatic cells with only some degrees of swelling and degeneration. (c \& d) Liver sections of GL pretreated rats before DIC treatment showing normal appearance of hepatocytes. $\mathrm{P}$, portal tract; $\mathrm{CV}$, central area; original magnification X 200 in (b \& d) and X 400 in (a \& c); $\mathrm{H} \& \mathrm{E}$.

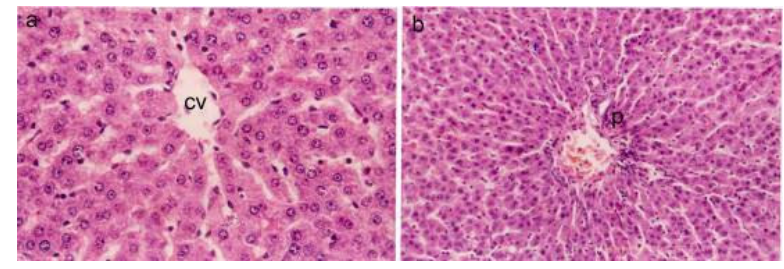

Fig. 8. Liver section of rat treated with DIC+MO showing normal appearance of hepatic cells showing better preserved appearance of hepatocytes as compared to the DIC-treated group ; original magnification X 200 in (b) and X 400 in (a); H\&E.

GSH is the most important endogenous antioxidant marker for chemical-induced toxicity to help eliminating the over produced ROS. In the present study, the level of GSH was markedly elevated in normal rats after treatment of CL. This result is consistent with previous studies which indicated that curcumin increased the GSH content in liver of tested animals [53; 54]. The observed increase in GSH content in normal rats could be attributed to the decrease GSH consumption or increase synthesis under the effect of antioxidant action of CL.

In addition to its well-known antioxidant activity, Curcumin may also enhance the detoxification process of DIC which in turn may contribute to the hepatoprotective effect of CL. Curcumin has been shown to increase GSH linked detoxification enzymes such as glutathione-S-transferase in rat liver [53; 55]. 
The induction of GSH linked enzymes has been suggested as a possible mechanism of the protective of curcumin [54]. The inhibition of enzymes such as cytochrome- P450 may be, at least in part, one of the possible modes of chemoprotective action of curcumin. In this respect, tumeric and curcumin have been shown to inhibit cytochrome- P450 enzymes both in vitro and in vivo [56].

GL extract protected against DIC-induced liver damage before DIC treatment also attenuated liver damage induced by DIC treatment as shown by low histopathological changes and ameliorated serum ALT activity in comparison to the DIC-treated group. GL and its flavonoids have been shown to produce hepatoprotection against a variety of hepatotoxic agents including $\mathrm{CCl} 4$ [26; 27], and aflatoxicn B1 [28]. Antihepatotoxicity of GL and its isoflavons reported to be due to their antioxidative action [29; 30]. Licorice contains substantial quantities of phenolic antioxidants (mostly in the form of pyranoisoflavan) some of which (e.g., Glabridin) are very potent antioxidant [29]. Licochalcone A, which is phenolic natural substance present in licorice, was demonstrated to have antioxidizing activity [29]. Results of the present study support this hyopthesis because the protective effect of GL is accompanied by normalization of MDA level and decreasing the depletion of total antioxidant capacity and antioxidant enzyme (SOD \& CAT) activities.

GSH play an important role in the detoxification of hepatotoxic substances [57]. GSH detoxification is mediated through the activation of GSH-transferase and formation of conjugation with toxic metabolites [57]. In the present work, the pretreatment with GL before DIC treatment induced elevation in hepatic GSH content; however, GL, by itself, did not affect its level in normal rats. Thus an increase in hepatic GSH content after GL pretreatment may represent a self defense mechanism in preventing hepatotoxicity and the elevation of GSH level with normal MDA level and may suggest that less toxic DIC metabolites were formed.

The hepatoprotective of GL may be due to its ability to block the bioactivation of DIC to toxic metabolites by decrease metabolite activation or enhance the detoxification of DIC. In this respect, glycyrrhetinic acid, the major biologically active ingredients of GL, has been shown to decrease the CCL4-induced liver injury by inhibiting the activity and expression of P450 enzymes [27]. GL treatment to male rats enhanced the glucuronidation pathway in rat livers [58]. Thus, GL not only plays important role by antioxidative effect, but also may play a role in reducing DIC toxicity.

The pretreatment with the MO extract significantly protected the animals from the liver injury and oxidative stress damage of DIC. Similarly, MO seed extract $(500 \mathrm{mg} / \mathrm{kg}$ b.wt) has been exhibited significant roles in protecting rats from arsenic-induced oxidative stress damage [59]. The antioxidant properties of MO in vivo and in vitro have been confirmed in the previous studies [36; 37]. Seeds of MO contain various antioxidants including tocopherols, vitamin $\mathrm{C}$, and glucosinolates [33; 40; 59]. Significant recovery in liver CAT and SOD activities as well as total antioxidant capacity after pretreatment with MO could be due to its antioxidant properties. In addition to its antioxidant effect, MO found to induce increase in the level of hepatic phase 1 and phase 2 detoxified enzymes [34]. Therefore, the observed hepatoprotective effect of MO may be attributing, at least in part, to its ability to decrease metabolic activation of DIC.

In conclusion, the results of this study demonstrated that CL, GL, and MO crude extracts protect against the DIC-induced liver damage in rats. These results show that the protective effect of the three extracts may be to their antioxidant activities and due to their ability to decrease the metabolic activation of DIC. The present results could lead to a possible development of a therapeutic remedy to limit or at least alleviate some of the reported DIC-induced side effects in human.

\section{ACKNOWLEDGEMENTS}

I thank Prof. Mohammed Badawey and Prof. Rokaya Mahmoud (Biology Dept. Head, National Organization of Drug Control and Research) for virtually making all biology facilities available for me to use.

\section{REFERENCES}

1. 1. Aydin, G., A. Gokcimen, M. Oncu, E. Cicek, N. Karahan, and O. Gokalp. 2003. Histopathologic changes in liver and renal tissues induced by different doses of diclofenac sodium in rats. Turk.J.Vet. Anim. Sci. 27:1131-1140.

2. Ouellette, G., B. Slitzky, J. Gates, S. Lagarde, and A. West. 1991. Reversible hepatitis associated with diclofenac. J clin Gastroenterol 13(2):205-210.

3. Hackstein, H., W. Mohl, W. Puschel, A. Stallmach, and M. Zeitz. 1998. Diclofenac-associated acute cholestasis hepatitis. Z.Gastroenterol 36(5):385-389.

4. Cantoni, L., R. Valaperta, X. Ponsoda, J. V. Castell, D. Barella, M. Rizzardini, A. Mangolini, L. hauri, and P. Villa. 2003. Induction of hepatic hem oxygenase- 1 by diclofenac in rodents : role of oxidative stress and cytochrome P-450 activity. J Hepatol 38:776-783.

5. Amin, A., and A. A. Hamza. 2005. Oxidative stress mediates drug-induced hepatotoxicity in rats:a possible role of DNA fragmentation. Toxicology 208:367-375

6. Gill, M., M. C. Ramirez, M. C. Terencio, and J. V. Castell. 1995. Immunochemical detection of protein adducts in cultured human hepatocytes exposed to diclofenac. Bioch Biophysic Acta 1272(3):140-146.

7. Tang, W., R. A. Stearns, S. M. Bandiera, Y. Zhang, C. Rabb, M. P. Braun, D. C. Dean, J. Rang, K. H. Leung, G. A. Doss, J. Strauss, G. Kwei, T. Rushmore, S. Chiu, and T. Baillie. 1999. Studies on cytochrome P-450-mediated bioactivation of diclofenac in rats and in human hepatocytes:identification of glutathione conjugated metabolites. Drug Metab Dispos $27(3): 365-372$. 
8. Tang, W. 2003. The metabolism of diclofenacenzymology and toxicology perspectives. Curr Drug Metab 4(4):319-329.

9. Galati, G., S. Tafazoli, O. Sabzevari, T. Chan, and B. O, PJ. 2002. Idosyncratic NSAID drug induced oxidative stress. Chem Biol Interact 142((1-2)):25-41.

10. Masubuchi, Y., S. Nakayama, and T. Horie. 2002. Role of mitochondrial permeability transition in diclofenac-induced hepatocyte injury in rats. Hepatology 35(3):544-551.

11. Gomez-Lechon, M., X. Ponsoda, E. O Connor, T. Donato, J. V. Castell, and R. Jover. 2003. Diclofenac induces appoptosis in hepatocytes by alteration of mitochondrial function and generation of ROS. Bioch Pharmacol 66(11):2155-2167.

12. Bort, R., X. Ponsoda, R. Jover, M. Gomez-Lechon, and J. V. Castell. 1999. Diclofenac toxicity to hepatocytes : a role for drug metabolism in cell toxicity. J pharm ExpTher 288(1):65-72.

13. Soobrattee, M. A., V. S. Neergheen, A. LuximonRamma, O. I. Aruoma, and T. Bahorun. 2005. Phenolic as potential antioxidant theraputic agents:Mechanism and actions. Mut.Res. 579:200-213.

14. Miquel, J., A. Bernd, J. M. Sempere, J. DiazAlperi, and A. Ramirez. 2002. The curcuma antioxidants:phaemacological effects and prospects for future clinical use. A review. Arch Geronto Geriatr 34:37-46.

15. Asia, A., and T. Miyazawa. 2001. Dietary curcuminoids prevent high-fat diet-induced lipid accumulation in rat liver and epididymal adipose tissue. J Nutr 131:2932-2935.

16. Soni, K. B., A. Rajan, and R. Kuttan. 1992. Reversal of aflatoxin induced liver damage by tumeric and curcumin. Cancer Lett 66(2):115-121.

17. Subramanian, L., and R. Selvam. 1999. Prevention of CCL4-induced hepatotoxicity by aqueous extract of turmeric. Nutr. Res. 19(3):429-441.

18. Park, E., C. H. Jeon, G. Ko, J. Kim, and D. Sohn. 2000. Protective effect of curcumin in rat liver injury induced by carbon tetrachloride. J.Pharm.Pharmacol. 52:437-440.

19. Nanji, A. A., K. Jokelainen, G. L. Tipoe, A. Rahemtulla, P. Thomas, and A. J. Dannenberg. 2003. Curcumin prevents alcohol-induced liver disease in rats by inhibiting the expression of NFKappa B-dependent genes. AmrJ Phsiol Gastrointest Liver Phsiol 284(2):G321-327.

20. Ramirez, M. C., M. D. Mesa, M. C. Aguilera, J. L. Quiles, L. Baro, C. L. Ramirez-Tortosa, E. Martinez-Vicyoria, and A. Gill. 1999. Oral administration of a tumeric extract inhibits LDL oxidation and has hypocholesterolemic effects in rabbits with experimental atherosclerosis. Atherosclerosis 147:371-378.

21. Chuang, S. E., A. L. Cheng, J. K. Lin, and M. L. Kuo. 2000. Inhibition by curcumin of diethynitrosamine-induced hepatic hyperplasia, inflammation,cellular gene products and cell-cyclerelated protein in rats. Food .Chem Toxicol 38(11):991-995.

22. Busquets, S., N. Carbo, V. Almendro, M. T. Quiles, F. J. Lopez-Soriano, and J. M. Argiles. 2001. Curcumin, a natural product present in tumeric, decreases tumor growth but does not behave as anticachetic compound in a rat model. Cancer Lett 167(1):33-38.
23. Lupper, S. 1999. A review of plants used in treatment of liver disease. a part two. Altern. Med. Rev. 4:179-189.

24. Wang, Z. Y., and D. W. Nixon. 2001. Licorice and cancer. Nutr. Cancer 39:1-11.

25. Shibata, S. 2000. A drug over the millennia: pharmacognosy, chemistry, and pharmacology of licorice. . Yakugaku. Zasshi. 120(10):849-862.

26. Wang, J. W., J. S. Guo, S. L. Liu, and M. A. Zern. 1998. Inhibitory effect of glycyrrhizin on NFkappa B binding activity in CCL4-plus ethanolinduced liver cirrhosis in rats. Liver 18(3):180-185.

27. Jeong, H., H. J. You, S. J. Park, A. E. Moon, Y. C. Chung, S. K. Kang, and H. K. Chun. 2002. Hepatoprotective effects of 18B-Glycyrrhetinic acid on carbon tetrachloride-induced liver injury: inhibition of cytochrome P 4502 E1 expression. Pharmaco. Res. 46(3):221-227.

28. Chan, H. T., C. Chan, and J. W. Ho. 2003. Inhibition of glycyrrhizic acid on aflatoxin B1induced cytotoxicity in hepatoma cells. Toxicolgy 188:211-217.

29. Vaya, J., P. A. Belinky, and M. Aviram. 1997. Antioxidant constituents from licorice roots: isolation, structure elucidation and antioxidative capacity toward LDL oxidation. Free Radic. Biol. Med. 23(2):302-313.

30. Kolbe, L., J. mmeyer, J. Batzer, U. Wensorra, K. T. Dieck, C. Mundt, R. Wolber, F. Stab, U. Schonrock, R. I. Ceilley, and H. Wenck. 2006. Anti-inflammatory efficacy of Licochalcone A: correlation of clinical potency and in vitro effects. . Arch Dermatol. Res. 298(1):23-30.

31. Ghasi, S., E. Nwobodo, and J. O. Ofili. 2000. Hypocholesterolemic effects of crude extract of leaf of Moringa oleifera Lam in high-fat diet fed wistar rats. J. Ethnopharmacol 69:21-25.

32. Caceres, A., A. Saravia, S. Rizzo, L. Zabala, E. De Leon, and F. Nave. 1992. Pharmacologic properties of Moringa oleifera.2:screening for antispasmodic, antiinflammatory and diuretic activity. J. Ethnopharmacol 36(3):233-237.

33. Guevara, A. P., C. Vargas, H. Sakurai, Y. Fujiwara, K. Hashimoto, T. Maoka, M. Kozuka, Y. Ito, H. Tokuda, and H. Nishino. 1999. An antitumor promoter from Moringa oleifera Lam. Mutat. Res. 440(2):181-188.

34. Bharali, R., J. Tabassum, and M. R. Azad. 2003. Chemomodulatory effect of Moringa oleifera Lam on hepatic carcinogen metabolising enzymes, antioxidant parameters and skin papillomagenesis in mice. Asian Pac.J.Cancer Prev. 4(2):131-139.

35. Kar, A., B. K. Choudhary, and N. G. Bandyopadhyay. 2003. Comparative evaluation of hypoglycaemic activity of some Indian medicinal plants in alloxan diabetic rats. J. Ethnopharmacol 84(1):105-108. 
36. Kumar, A., and L. Pari. 2003. Antioxidant action of Moringa oleifera Lam.(drumstick) against antitubercular drugs induced lipid peroxidation in rats. J.Med.Food 6(3):255-259.

37. Siddhuraju, P., and K. Becker. 2003. Antioxidant properties of various solvent extracts of total phenolic constituents from three different agroclimatic origin of drumstick tree (Moringa olieifera Lim.) leaves. J.Agric.Food Chem. 51(8):2144-2155.

38. Murakami, A., Y. Kiyazono, S. Jiwajinda, K. Koshimizu, and H. Ohigashi. 1998. Niaziminin, a thiocarbamate frome the leaves of Moringa oleifera holds a strict structural requirement for inhibition of tumor-promoter-induced Epstein-Barr virus activation. Planta . Med. 64(4):319-323.

39. Lalas, S., and J. Tsaknis. 2002. Characterization of Moringa oleifera seed oil variety. J.Food Compos.Analy. 15:65-77.

40. Bennet, R. N., F. A. Mellon, N. Foidl, J. H. Pratt, M. S. Dupont, L. Perkins, and P. A. Kroon. 2003. Profiling glucosinolates and phenolics in vegetative and repductive tissues of the multi-purpose tress Moringa oleifera L.(horseradish tree) and Moringa stenopetala L. J.Agric.Food Chem. 51(12):35463453.

41. Pan, X., H. Liu , G. Jia, and Y. Y. Shu. 2000. Microwave-assisted extraction of glycyrrhizic acid from licorice root. Bioch Enginer. J. 5:173-177.

42. Paget, G. E., and R. Thomson. 1979. Standard Operating Procedures in Toxicology. M T P Press limited, Lancaster. England.

43. Van Dooran, R., C. M. Leijdekkers, and P. T. Henderson. 1978. Synergistic effects of phorone on the hepatotoxicity of bromobenzene and paracetamol in mice. Toxicol 11:225-233.

44. Packer, L., and E. Cadenas. 2002. Oxidative stress and disease. In E. Cadenas and L. Packer, editors. Handbook Of Antioxidants. Marcel Dekker,Inc., New Yok,Basel,USA. 5-8.

45. Uchiyama, M., and M. Mihara. 1978. Determination of malonaldehyde precursor in tissues by thiobarbituric acid test. Anal Biochem 86:271-278.

46. Sun, M., and S. Zigman. 1978. An improved spectrophotometric assay for superoxide dismutase based on epinephrine autoxidation. Anal Biochem 247(10):81-89.

47. Aebi, H. 1984. Catalase in vitro. Meth Enzymol. 121-26.

48. Benzie, I. F., and J. J. Strain. 1996. The ferric reducing ability of plasma (FRAP) as a measure of ( antioxidant power): The FRAP assay. Anal Biochem 293(1):70-76.
49. Reitman, S., and S. Frankel. 1957. A colorimetric method for the determination of serum glutamic oxalacetic and glutamic pyruvic transaminases. Am J Clin Path 28:56 -63.

50. Peterson, G. L. 1977. A simplification of the protein assay method of lowry et al which is more generally applicable. Anol Biochem 83:346 -356.

51. Djordjevic, V. B. 2004. Free radicals in cell biology. Int Rev.Cytology 237:57-89.

52. Akila, G., V. Rajakrishnan, P. Viswanathan, K. N. Rajashekaran, and V. P. Menon. 1998. Effects of curcumin on lipid profile and lipid peroxidation ststus in experimental hepatic fibrosis. Hepatology Res 11:147-157.

53. Piper, J. T., S. S. Singhal, S. S. Mohammad, R. T. Torman, Y. C. Awasthi, and A. S. Awasthi. 1998. Mechanisms of anticarcinogenic properties of curcumin: the effect of curcumin on glutathione linked detoxification enzymes in rat liver. Inter $\mathrm{j}$ Biochem.Cell. Biol. 30:445-456.

54. Iqbal, M., S. D. Sharma, Y. Okazaki, M. Fujisawa, and S. Okada. 2003. Dietary supplementation of curcumin enhance antioxidant and phase II metabolizing enzymes in ddY male mice: pssible role in protection against chemical carcinogenic and toxicity. Parmacol \& Toxicol 92:33-38.

55. Sharma, R. A., C. R. Ireson, R. D. Verschole, K. A. Hill, M. L. Williams, C. Leuratti, M. M. Manson, L. J. Marnett, W. P. Steward, and A. Gescher. 2001. Effects of dietary curcumin on glutathione stransferase and malondialdehyde-DNA adducts in rat liver and colon mucosa: relatioship with drug levels (1). Clin Cancer Res 7(5):1452-1458.

56. Thapliyal, R., and G. B. Maru. 2001. Inhibition of cytochrome P450 isozymes by curcumins in vitro and in vivo. Food .Chem Toxicol 39(6):541-547.

57. Rana, S. V., T. Allen, and R. Singh. 2002. Inevitable glutathione, then and now. Indian J. Exp. Biol. 40(6):706-716.

58. Moon, A., and S. H. Kim. 1997. Effect of Glycyrrhiza glabra root and glycyrrhizin on the glucuronidation in rats. Plant Med. 63(2):115-119.

59. Gupta, R., G. M. Kannan, M. Sharma, and S. J. S. Flora. 2005. Theraputic effects of Moringa oleifera on arsenic-induced toxicity in rats. Environ. Toxicol.Pharmacol. 